\title{
Review
}

\section{General principles for risk assessment of living modified organisms: Lessons from chemical risk assessment ${ }^{1}$}

\author{
Ryan A. HILL and Cyrie SENDASHONGA \\ Biosafety Programme, Secretariat of the Convention on Biological Diversity, 393 St Jacques St, Suite 300, Montreal, \\ Quebec, H2Y1N9 Canada
}

\begin{abstract}
Modern biotechnology has led to the development and use of Living Modified Organisms (LMOs) for agriculture and other purposes. Regulators at the national level are increasingly depending on risk assessment as a tool for assessing potential adverse effects of LMOs on the environment and human health. In addition, the Cartagena Protocol on Biosafety, an international agreement expected to enter into force in the near future, requires risk assessment as the basis for decision-making regarding import of some LMOs. While LMO risk assessment is relatively new, there are other risk assessment disciplines which have developed over longer time periods. The field of assessment of the environmental and human health risks of chemicals is particularly well developed, and is similar in application to LMO risk assessment. This paper aims to draw lessons for LMO risk assessment from the vast experience with chemical risk assessment. Seven general principles are outlined which should serve as a useful checklist to guide assessments of risks posed by LMOs.
\end{abstract}

Key words: risk assessment / living modified organisms / genetically modified organisms / biosafety protocol

\section{INTRODUCTION}

The term "risk assessment" is used to describe a wide range of formal and informal methodologies in a range of disciplines. Most commonly, risk assessments attempt to characterize potential adverse impacts associated with particular activities or events. One discipline in which risk assessment is applied is the characterization of potential adverse effects on the environment or human health posed by Living Modified Organisms ${ }^{2}$ (LMOs), commonly referred to as Genetically Modified Organisms. Of particular interest in recent years are potential risks associated with the use and release of genetically modified crops. Risk assessment of LMOs is a relatively young field in comparison to other risk assessment applications. However, the recent growth in development of biotechnology has generated unprecedented interest in the potential positive and negative impacts of such technology. Regulators are increasingly depending on risk assessment as a tool in supporting decision-making in the field of biotechnology.

Most importantly, the Cartagena Protocol on Biosafety to the Convention on Biological Diversity, which was adopted in January 2000 and is expected to enter into force in the near future, will require governments to conduct risk assessments in support of decisions regarding the import of LMOs including genetically modified crops. Annex III of the Biosafety Protocol outlines a broad framework for risk assessment of LMOs by defining some general principles

\footnotetext{
* Corresponding author:

Fax: 514-288-6588; e-mail: ryan.hill@biodiv.org / cyrie.sendashonga@biodiv.org

1 The views expressed in this paper are those of the authors and do not represent views of the Secretariat of the Convention on Biological Diversity or the Parties to the Convention.

2 The term "Living Modified Organism" is defined in the Cartagena Protocol on Biosafety to the Convention on Biological Diversity as any living organism that possesses a novel combination of genetic material obtained through the use of modern biotechnology. The definitions for "Living Modified Organism", "Living Organism" and "Modern Biotechnology" contained in the Cartagena Protocol on Biosafety and used in many international fora are specific, and may not be exactly equivalent to common definitions of similar terms such as genetically modified organism.
} 


\section{R.A. Hill and C. Sendashonga}

and elements of methodology. The points covered in Annex III will be a useful starting point for governments. However, the complexity of risk assessment necessitates more detailed guidance, and it is in this regard that lessons from other fields of risk assessment may prove invaluable.

Formal risk assessment methodologies exist in a range of disciplines, including business and management (Vatter et al., 1978), insurance (see the Journal of Risk and Insurance), fisheries management (Francis, 1992) and conservation biology (Maguire, 1991). In the context of assessing potential risks associated with LMOs, however, there is one field of study which is particularly likely to provide insights regarding approaches to risk assessment. This is the field of the assessment of ecological and human health risks from chemical contaminants. Risk assessment for chemicals has benefited from immense effort and funding over the past 20 years, especially in the United States (e.g., the Superfund program; see Hamilton and Viscusi, 1994) but also in Canada, Europe and Australia. In addition, assessment of risks from chemicals is in many ways conceptually similar to the assessment of risks from LMOs. For these reasons, it is worthwhile to look at the experiences in this field in an attempt to discern some key principles for approaching LMO risk assessment.

Assessment of human health and ecological risks from chemicals and other stressors dates back at least to the 1970s (see Suter, 1993, for further reading). In 1983 the National Research Council in the United States defined a formal set of steps involved in human health risk assessment (NRC, 1983). This "Red Book" formed the basis of the widespread paradigm for risk assessment which has evolved and is used today. The general approach outlined in that publication formed the basis for development of guidance on ecological risk assessment, and more specific guidance on human health risk assessment. Subsequent development of risk assessment frameworks in the United States, Canada and elsewhere took place throughout the 1980s and 1990s (Barnthouse, 1994; NRC, 1993). Examples of guidance documents include the Guidelines for Ecological Risk Assessment (EPA, 1998) developed by the United States Environmental Protection Agency; the Framework for Ecological Risk Assessment (CCME, 1996) developed by the Canadian Council of Ministers of the Environment for application to contaminated sites; the European Commission Regulation on risk assessment for existing substances (CEC, 1996), and the UK's Guidelines for Environmental Risk Assessment and Management (DEFRA, 2000).
Under this broad risk assessment paradigm, there were some key steps held in common. These can be summarized as follows:

- Problem formulation.

- Assessment of case-specific exposure to a stressor (e.g., likelihood of exposure or of particular levels of exposure).

- Assessment of the relationship between level of exposure and magnitude of associated effects.

- Overall characterization of risks in terms of the likelihood and magnitude of effects.

Risk assessment frameworks vary considerably in the number and definition of particular steps and in the terminology used to describe these steps. The important aspects are simply that any risk assessment begins with identification and formulation of the problem, usually in the context of a decision (NRC, 1996), and that the concept of risk has two main components, consisting of the relative likelihood or probability of potential adverse effects, and the magnitude or consequences of those effects.

Many risk assessment frameworks, including those developed by the USEPA, were developed for application to risks posed by a range of stressors with potential adverse ecological or human health effects. In practice, most applications of risk assessment have addressed chemical stressors, ranging from metals to persistent organic pollutants (Suter, 1993). However, the frameworks have been applied successfully to integrated assessments of the risks posed to ecological systems by a range of chemical, physical, and biological stressors.

There is general agreement that the broader principles of this risk assessment paradigm are relevant to risk assessment of LMOs (EC, 1998; ERMA, 1999; Fiksel and Covello, 1986; Kjellsson, 1997; Miller et al., 2002; Nickson and McKee, 2002; Suter, 1993), although it is recognized that the details must be different (Kappeli and Auberson, 1998; Strauss, 1991). Case studies of LMO risk assessments that follow the conventional frameworks exist (OSTP, 2001; Sears et al., 2001). In addition, many regulatory agencies have guidance for LMO risk assessment that is in accordance with conventional risk assessment approaches at a broad level, such as the UK's guidance on principles of risk assessment and monitoring for the release of genetically modified organisms (DETR, 1999), the New Zealand Environmental Risk Management Authority's technical guidance for the application of risk assessment under the Hazardous Substances and New Organisms Act (ERMA, 1999; 2000a; 2000b), and the European Commission's 
guidance notes supplementing Annex II to Directive 2001/18/EC of the European Parliament and of the Council on the deliberate release into the environment of genetically modified organisms (EC, 2002).

While many LMO risk assessments have attempted to build on existing risk assessment frameworks, some of the important lessons regarding how to conduct risk assessment have not been recognized in any consistent manner. This paper aims to discern a few general principles for risk assessment from the vast experience accumulated in the field of chemical risk assessment, and to relate these to the context of risk assessment for LMOs. These principles are not expected to prescribe particular methodologies or to predefine the scope of risk assessments. Rather, this paper is intended simply to elucidate some key principles that could serve as a useful checklist to guide assessments of risks posed by LMOs.

\section{LESSON 1: ACCOUNT FOR UNCERTAINTY}

Uncertainty is inherent in the concept of risk. Sources of uncertainty include lack of knowledge about a system or process, measurement errors, and random variability or stochasticity (Finkel, 1990; Morgan and Henrion, 1990; Suter, 1990). There is also variability in characteristics of individuals within a human, animal or plant population. Taken together, the various sources of uncertainty in the assessment of risks posed by chemicals can be several orders of magnitude (Bogen, 1994; Burmaster and Harris, 1993; Gaylor et al., 1993).

In the chemical risk assessment literature, extensive efforts have been made at developing methods of incorporating uncertainty into assessments in a way that is useful to decision-makers. Methods range from development of alternative scenarios (in the case of qualitative assessments) to simple sensitivity analyses to detailed probabilistic models that explicitly account for uncertainty and natural variability (Burmaster and Anderson, 1994; DEFRA, 2000; EPA, 1998; Finkel, 1990; Finley and Paustenbach, 1994).

A key general principle emerging from the chemical risk assessment literature is that better decisions can be made when uncertainty is incorporated into risk assessments, and that the best approaches for dealing with uncertainty depend on the decision-making context and the quality and quantity of data. Incorporating uncertainty into assessments has been shown quantitatively to enable improved decision-making regarding management of chemical contaminants (Begley, 1996; Dakins et al., 1994; Finkel, 1990; Morgan and Henrion, 1990; Reckhow, 1994). A major advantage of explicitly accounting for uncertainty is that it allows decision-makers to make decisions based on the actual data and information. The alternative, where risk assessors select point values, is much less informative, and in cases where conservative estimates are used, the compounded effect of conservative assumptions can give severely biased estimates of risk (Cullen, 1994).

Scientists and regulators involved in assessing risks associated with LMOs are fully aware of the large amount of scientific uncertainty regarding potential ecological and human health effects (e.g., Edmonds Institute, 1998; ERMA, 2000a). Indeed, uncertainties regarding risks posed by LMOs may often be more difficult to deal with than uncertainties regarding risks posed by chemical contaminants (EC, 1998; Strauss, 1991). The Cartagena Protocol on Biosafety explicitly recognizes that scientific uncertainties exist and that decisions must be taken recognizing that those uncertainties may not be resolved.

In spite of this recognition of the importance of uncertainties, approaches for dealing with uncertainty in LMO risk assessment have not yet evolved as in other fields. In the case of LMOs as with chemicals, poor data and confounding factors generally make it difficult to detect ecologically meaningful relationships between a stressor (e.g., an LMO) and ecological variables, even if they exist (Edmonds Institute, 1998). In statistical terms, the possibility of type II errors is large, because the statistical ability or "power" to detect significant relationships is often low (see Peterman, 1990, for a discussion of statistical power). However, while hypothesis testing and consideration of statistical power may be important in some contexts, such approaches are not the most useful for decision-making. For decisionmakers, it is not so important to know whether a relationship is statistically significant or not, rather it is appropriate to consider the relative likelihoods of different possible relationships. For example, if data suggest that a link may exist between ingestion of an LMO crop and an adverse effect in an animal population, but there are not enough data to show a statistically significant relationship, would it be reasonable to assume that the relationship does not exist? Of course not, but that is the usual quantitative outcome of a standard statistical test, because the null hypothesis of no relationship cannot be rejected. More appropriate methods for addressing uncertainties in a decisionmaking context, such as those based on assessment of likelihoods or using Bayesian methods, have been utilized for many years in other risk assessment fields related to management of chemicals and fisheries 


\section{R.A. Hill and C. Sendashonga}

(Ellison, 1996; Hill, 1996; Punt and Hilborn, 1997; Thompson, 1992). These methods are equally well-suited to LMO risk assessment and should be seriously considered in all cases where data are highly uncertain. Simply stating that data are uncertain and concluding that decisions should be precautionary (or vice-versa) is poor use of scientific information and of little use to decisionmakers.

\section{LESSON 2: CONSIDER MULTIPLE LINES OF EVIDENCE}

Some fields of chemical risk assessment are particularly fraught with uncertainties that are difficult to resolve. For example, estimation of human health risks depends in large part on extrapolation of laboratory dose-response data from animals to humans, and extrapolation of effects at high doses to effects at low doses (Gaylor et al., 1993). In these cases, use of all possible data sources is particularly desirable because of the enormous uncertainty in the extrapolations. For example, there may be epidemiological data resulting from accidental exposures of people (e.g., as for methylmercury; see Clarkson, 1990). Combined with lab data from animal studies, these two data sources become complementary sources of evidence in a risk assessment.

Similarly for ecological risk assessment, significant uncertainties have led scientists to adopt methods that use multiple lines of evidence. For example, effects of sediment contamination on marine benthic communities typically relies on a wide variety of data sources such as comparison of contaminant concentrations to benchmark data, case-specific toxicity tests, and analyses of species abundance and community structure (Chapman et al., 1996; Ingersoll et al., 1997). The USEPA guidelines for risk assessment stress the importance of considering all lines of evidence (EPA, 1998), and at least one procedure has been developed for evaluating risks based on the formal weighting of evidence from multiple data sources (Menzie et al., 1996).

In the context of LMO risk assessment, uncertainties are at least as pervasive as with chemicals. For this reason, LMO risk assessments often rely on multiple types of data ranging from laboratory investigations (e.g., molecular genetic analyses of phenotypic properties, microcosm and mesocosm experiments), field trials, computer simulations of dispersal and gene flow, etc. (Edmonds Institute, 1998; NRC, 2002). Some authors have explicitly noted the need to consider these various types of information together when making judgments about risks (Nickson and McKee, 2002; Strandberg et al.,
1998). In this regard, the most important message from the chemical risk assessment literature is to consider all types and sources of data, even those with less certainty than others - few data are better than no data.

\section{LESSON 3: ASSESS RISKS IN A COMPARATIVE CONTEXT}

Regulators and scientists involved in LMO risk assessment have embraced the concept of comparative risk assessment due to the complexity of LMO risk assessment. For example, various risk assessment frameworks, including Annex III of the Cartagena Protocol on Biosafety, refer to the importance of assessing risks posed by LMOs in the context of the risks posed by non-modified recipients or parental organisms in the receiving environment (NRC, 2002; OGTR, 2001; UNEP, 1995). One important component of comparative risk assessment for LMOs has been the concepts of substantial equivalence and familiarity. The Canadian Food Inspection Agency (CFIA, 2001) and others evaluate some LMOs in part by testing to determine if they have the same characteristics as nonLMO counterparts. If the important characteristics are the same, LMOs are treated from the regulatory perspective as equivalent to non-LMO counterparts. This type of comparative approach may be restricted to comparing LMOs to non-LMO counterparts, or may be applied more broadly to account for differences in cropping systems or other aspects of production (NRC, 2002). While the details of what quantitative criteria constitute substantial equivalence or familiarity can be debated, the concept is rooted in the logic of comparative risk assessment, whereby decisions are supported by comparative assessments of risk.

Experience from the chemical risk assessment literature suggests that there are a couple of points that should be emphasized with respect to comparative risk assessment. First, it is important to consider a wide range of alternatives from the start of an assessment, as discounting certain alternatives prematurely is a common source of poor decision-making. Second, it is useful to focus a risk assessment by explicitly characterizing the questions that need to be answered to support a particular management decision. In general, it is important to view risk assessments primarily as a tool in decision-making rather than as a means of scientific research (Mathes and Winter, 1993; McCarty, 1997; Power and McCarty, 1997). Finally, beyond the scale of a particular LMO risk assessment, it should be noted that the concept of 
comparative risk assessment can be used to evaluate the relative risks posed by a range of environmental problems (e.g., LMOs, chemicals, etc.), and to use that information to set priorities for addressing those problems.

\section{LESSON 4: BE FLEXIBLE REGARDING THE LEVEL OF DETAIL FOR RISK ASSESSMENTS}

Experience in chemical risk assessment has shown that it is very difficult to predetermine the level of detail appropriate for a risk assessment, for at least three reasons. First, it is difficult to know beforehand whether risks can be ruled out based on a simplified initial assessment or whether more detailed assessment will be warranted. In other words, findings of initial assessments may lead to obvious conclusions, or further assessment may be necessary. Second, the level of detail appropriate for a risk assessment depends on the nature of the decision to be supported. For example, if a preliminary analysis shows that a potential risk pathway is unimportant, there is no need to investigate that pathway in more detail. Finally, the level of detail for a particular assessment can be limited by the type and quality of data that are available.

In recognition of these factors, many risk assessment frameworks in North America and elsewhere refer to the need for initial or "scoping-level" assessments as the first step, followed by a detailed assessment if necessary (CCME, 1996; Cura, 1998; DEFRA, 2000; EPA, 1998). In this way, many risk assessments are considered to be "tiered", meaning that they increase in complexity depending on the findings at each level of assessment (Hill et al., 2000).

Some LMO risk assessments and frameworks have suggested a tiered approach in which the level of detail increases depending on the results of the previous tier (EC, 1998; ERMA, 2000b; Kjaer et al., 1999; Strandberg et al., 1998). The Cartagena Protocol on Biosafety recognizes that the information required for any particular risk assessment will vary in nature and in level of detail depending on many factors. Still, there is not yet a widespread understanding of the concept of a tiered approach and how it relates to the level of detail of an assessment. What is important is not whether there is a pre-determined number of steps or a particular methodology, but rather that risk assessors understand that it is appropriate to increase the level of detail of any assessment depending on the results of preliminary analyses, the nature of the decision to be supported, and the limitations of available data.

\section{LESSON 5: RISK ASSESSMENTS SHOULD BE ITERATIVE AND ADAPTIVE}

The need for an iterative and adaptive approach to risk assessment is critical. These concepts appear in the LMO risk assessment literature but have not yet been fully defined or elaborated. Risk assessment must be "iterative" because some details of an assessment may need to be revisited depending on the findings of other components of the assessment (e.g., if an assumption is shown to be incorrect) (NRC, 1996). This is a different point from the need for tiered assessments and increasing level of detail as discussed above (see lesson 4). Some authors have emphasized the need for iterative assessments in the context of LMO risk assessment (DEFRA, 2000; Nickson and McKee, 2002).

Risk assessments should also be "adaptive". Adaptive management is a cornerstone of science-based management and decision-making (Walters, 1986). Some LMO risk assessment frameworks acknowledge that risk assessments should be subject to review when new data are made available (EC, 2001; DETR, 1999; OGTR, 2001). However, there is much more to adaptive management than re-evaluating risks when new data become available. Risk assessments must be adaptive to changes in any parameters or assumptions in the assessment, including baseline ecological conditions or the context of the decision in which assessments are carried out (Harvey et al., 1995). For example, over the long-term, shifts in ecological conditions may change the potential for adverse effects from LMOs (e.g., a weedy relative of a GM crop may establish a population in an area that was previously not habitable). Adaptive management infers that management decisions should be re-evaluated whenever there have been changes that affect risk estimates, or whenever management actions generate new empirical data, and implies that monitoring to enable detection of such changes is an integral part of management.

\section{LESSON 6: FORMALLY EVALUATE THE EXPECTED VALUE OF ADDITIONAL INFORMATION}

For any decision, there is usually an alternative to delay the decision and to collect more information, or to take an interim decision pending collection of more data. In considering this alternative, assessing the expected value of additional information helps us to evaluate, for any particular risk assessment, whether to take a decision now or whether to get more information. This concept 


\section{R.A. Hill and C. Sendashonga}

has been discussed in the broader risk assessment literature and has been shown quantitatively to improve decision-making (Finkel, 1990; Finkel, 1994). Formal evaluations of the value of the information have been made in the context of chemical risk assessment to cases of acid rain policy (North and Balson, 1985) and remediation of contaminated sites (Dakins et al., 1996). A more generic explanation of methods is made by Morgan and Henrion (1990).

Uncertainties in risk assessment are large, and research to reduce uncertainties can be extremely expensive and time-consuming exercises, and in some cases it may not even be possible to reduce the uncertainties at all. Therefore, it is important to understand the contribution of different uncertainties to overall uncertainty in a risk assessment (McKone and Bogen, 1991), with the aim of addressing those for which the value of collecting new information, measured as the expected improvement in a decision through reduced uncertainty, is worth the cost of that information (Finkel, 1994).

In the context of LMO risk assessment, the same logic applies when considering collection of additional data to support decision-making. Data should not be collected simply to address the largest uncertainties, or those which are most easily resolved. Rather, data collection in support of LMO risk assessment should be based on formal evaluation of the expected value of those data in relation to their costs.

\section{LESSON 7: ASSESS CUMULATIVE EFFECTS AS PART OF RISK ASSESSMENT}

Cumulative effects refer to the combined impact of multiple stressors on human or ecological health. For example, we may want to understand not only the risks associated with exposure of an animal population to an LMO crop, but the combined effect of the LMO crop, pesticides, herbicides, and other stressors in the same geographic area. Cumulative effects can be considered in different ways, including modeling of scenarios and assessment of the likelihoods of alternative scenarios. Cumulative effects are important to consider because the combined effects of many stressors can be catastrophic even if the isolated effect of one stressor is low. Although consideration of the combined effects of multiple actions has occurred primarily under the related discipline of Environmental Impact Assessment (e.g., the Canadian Environmental Assessment Act; see Suter, 1993, for additional references and discussion), the USEPA has developed guidance on cumulative risk assessment (EPA, 1997), in recognition of the need to consider the cumulative effects of multiple stressors on human and ecological health. In addition, some risk assessors have promoted the idea of Regional Risk Assessments, which would aim in part to consider at once the cumulative risks posed by multiple sources of contaminants within a particular spatial region (see Suter, 1993, for discussion).

\section{CONCLUSION}

These seven lessons from chemical risk assessment do not form an exhaustive list of principles for risk assessment of LMOs. There are undoubtedly other principles that could be derived from other disciplines. Even from the LMO literature itself, for example, we can see from the much publicized case of the monarch butterfly that stakeholder involvement and deliberation in the risk assessment process is invaluable (PCT, 2002; Sears et al., 2001), and may be considered an important principle for risk assessment as has been suggested in the past (NRC, 1996).

It is not expected that it would be easy or even possible to develop a single risk assessment framework applicable for dealing with all LMO risk assessments. Indeed, some experts have already concluded that even within a particular field such as aquaculture, a single approach to risk assessment may not be possible due to the variability in the nature and scope of risks to be considered (Miller et al., 2002). However, for whatever particular methodologies are developed, the lessons discussed in this paper, gained from years of experience in the closely related field of chemical risk assessment, are likely to be a useful guide or checklist for scientists tasked with assessing the human health or ecological risks posed by LMOs.

Received September 19, 2002; accepted November 18, 2002.

\section{REFERENCES}

Barnthouse LW (1994) Issues in Ecological Risk Assessment: the CRAM Perspective. Risk Analysis 14: 251-256

Begley R (1996) Risk-based remediation guidelines take hold. Environ. Sci. Technol. 30: 438A-441A

Bogen KT (1994) A note on compounded conservatism. Risk Analysis 14: 379-381

Burmaster DE, Anderson PD (1994) Principles of good practice for the use of Monte Carlo techniques in human health and ecological risk assessments. Risk Analysis 14: 477-481

Burmaster DE, Harris RH (1993) The magnitude of compounding conservatism in Superfund risk assessments. Risk Analysis 13: 131-134 
Review: Principles for risk assessment of LMOs

CCME (Canadian Council of Ministers of the Environment) (1996) A Framework for Ecological Risk Assessment: General Guidance. Prepared by the CCME Subcommittee on Environmental Quality Criteria for Contaminated Sites

CEC (Commission of the European Communities) (1996)

Technical guidance documents in support of the Commission Directive 93/67/EEC on risk assessment for new substances and the Commission Regulation No. 1488/94 on risk assessment for existing substances. Brussels, Belgium

CFIA (Canadian Food Inspection Agency) (2001) Regulatory Directive Dir94-08: Assessing Criteria for Determining Environmental Safety of Plants with Novel Traits. Plant Health and Production Division, Plant Biosafety Office

Chapman P, Paine MD, Arthur AD, Taylor LA (1996) A triad study of sediment quality associated with a major, relatively untreated marine sewage discharge. Marine Pollution Bulletin 32: 47-64

Clarkson TW (1990) Human health risks from methylmercury in fish. Environ. Toxicol. Chem. 9: 957-961

Cullen AC (1994) Measures of compounding conservatism in probabilistic risk assessment. Risk Analysis 14: 389-393

Cura JJ (1998) Ecological risk assessment. Water Environ. Res. 70: 968-971

Dakins ME, Toll JE, Small MJ (1994) Risk-based environmental remediation: decision framework and role of uncertainty. Environ. Toxicol. Chem. 13: 1907-1915

Dakins ME, Toll JE, Small MJ, Brand KP (1996) Risk-based environmental remediation: Bayesian Monte Carlo analysis and the expected value of sample information. Risk Analysis 16: $67-79$

DEFRA (Department for Environment, Food and Rural Affairs) (2000) Guidelines for Environmental Risk Assessment and Management - Revised Departmental Guidance. United Kingdom

DETR (Department of the Environment, Transport and Regions) (1999) Guidance on principles for risk assessment and monitoring for the release of genetically modified organisms. DETR/ACRE guidance note 12, London, UK

EC (European Commission) (1998) Quantitative Environmental Risk Assessment for Genetically Modified Organisms. Institute for Health and Consumer Protection

EC (European Community) (2001) Directive 2001/18/EC of the European Parliament and of the Council, on the deliberate release into the environment of genetically modified organisms, 12 March 2001

EC (European Commission) (2002) Commission decision of 24 July 2002 establishing guidance notes supplementing Annex II to Directive 2001/18/EC of the European Parliament and of the Council on the deliberate release into the environment of genetically modified organisms and repealing Council Directive 90/220/EEC

Edmonds Institute (1998) Manual for Assessing Ecological and Human Health Effects of Genetically Engineered Organisms. Prepared by the Scientists' Working Group on Biosafety

Ellison AM (1996) An introduction to Bayesian inference for ecological research and environmental decision-making. Ecol. Appl. 6: 1036-1046

EPA (Environmental Protection Agency) (1997) Guidance on Cumulative Risk Assessment. Part 1. Planning and Scoping, Science Policy Council, EPA, Washington, D.C.

EPA (Environmental Protection Agency) (1998) Guidelines for Ecological Risk Assessment. EPA 630/R-95-002F, Washington, D.C.

ERMA (Environmental Risk Management Authority) (1999) Identifying risks for applications under the Hazardous Substances and New Organisms Act 1996, ER-TG-01-1 9/99, New Zealand

ERMA (Environmental Risk Management Authority) (2000a) Preparing information on risks, costs and benefits for applications under the Hazardous Substances and New Organisms Act 1996, ER-TG-03-1 7/00, New Zealand

ERMA (Environmental Risk Management Authority) (2000b) Assessment of effects of hazardous substances and new organisms on human health, ER-TG-02-1 1/00, New Zealand

Fiksel JR, Covello VT (1986) The suitability and applicability of risk assessment methods for environmental applications of biotechnology. In Fiksel JR, Covello VT, eds, Biotechnology Risk Assessment - Issues and Methods for Environmental Introductions. Permagon Press, pp 1-34

Finkel AM (1990) Confronting Uncertainty in Risk Management. Center for Risk Management, Resources for the Future, 1616 P Street, N.W., Washington, D.C.

Finkel AM (1994) Risk assessment research: only the beginning. Risk Analysis 14: 907-911

Finley B, Paustenbach D (1994) The benefits of probabilistic exposure assessment: three case studies involving contaminated air, water and soil. Risk Analysis 14: 53-73

Francis RICC (1992) Use of risk analysis to assess fishery management strategies: a case study using orange roughy on the Chatham Rise, New Zealand. Can. J. Fish. Aquat. Sci. 49: 922-930

Gaylor DW, Chen JJ, Sheehan DM (1993) Uncertainty in cancer risk assessments. Risk Analysis 13: 149-154

Hamilton JT, Viscusi WK (1994) Human health risk assessments for Superfund. Ecol. Law Q. 21: 573-610

Harvey T, Mahaffey KR, Velazquez S, Dourson M (1995) Holistic risk assessment: an emerging process for environmental decisions. Regul. Toxicol. Pharmacol. 22: 110-117

Hill RA (1996) From science to decision-making: the applicability of Bayesian methods to risk assessment. Human Ecol. Risk Assess. 2: 636-642

Hill RA, Chapman PM, Mann GS, Lawrence GS (2000) Level of detail in ecological risk assessments. Mar. Pollut. Bull. 40: 471-477

Ingersoll CG, Dillon T, Biddinger GR (1997) Ecological Risk Assessment of Contaminated Sediments. SETAC Press, Pensacola, FL, USA

Kappeli O, Auberson L (1998) How safe is safe enough in plant genetic engineering? Trends Plant Sci. 3: 276-281

Kjaer C, Damgaard C, Kjellsson G, Strandberg B, 


\section{R.A. Hill and C. Sendashonga}

Strandberg M (1999) Ecological risk assessment of genetically modified higher plants - identification of data needs. NERI Technical Report No. 303. Ministry of Environment and Energy, National Environmental Research Institute, Denmark

Kjellsson G (1997) Principles and procedures for ecological risk assessment of transgenic plants. In Kjellsson G, Simonsen V, Ammann K, eds, Methods for Risk Assessment of Transgenic Plants II - Pollination, Gene-Transfer and Population Impacts. Birkhauser Verlag, Basel, Switzerland, pp 221-236

Maguire LA (1991) Risk analysis for conservation biologists. Conserv. Biol. 5: 123-125

Mathes K, Winter G (1993) Ecological risk assessment and the regulation of chemicals: 3 . Balancing risks and benefits. Sci. Total Environ., supplement 1993: 1679-1687

McCarty LS (1997) Environmental risk assessment within a decision making framework. Environ. Toxicol. Chem. 16: 122-123

McKone TE, Bogen KT (1991) Predicting the uncertainties in risk assessment. Environ. Sci. Technol. 25: 1674-1681

Menzie C, Henning MH, Cura J, Findelstein K, Gentile J, Maughan M, Metchell D, Petron S, Potocki B, Sversky S, Tyler P (1996) Special report of the Massachusetts weightof-evidence workgroup: a weight-of-evidence approach for evaluating ecological risk. Human Ecol. Risk Assess. 2: 277-304

Miller LM, Kapuscinski AR, Senanan W (2002) A biosafety approach to addressing risks posed by aquaculture escapees. Proceedings of the Expert Consultation on Biosafety and Environmental Impact of Genetic Enhancement and Introduction of Improved Tilapia Strains/Alien Species in Africa, 20-23 February 2002, Nairobi, Kenya. Published by the International Centre for Living Aquatic Resources Management (ICLARM)

Morgan MG, Henrion M (1990) Uncertainty - A Guide to Dealing with Uncertainty in Quantitative Risk and Policy Analysis. Cambridge University Press

Nickson TE, McKee MJ (2002) Ecological assessment of crops derived through biotechnology. In Thomas JA, Fuchs FL, eds, Biotechnology and Safety Assessment, 3rd edn. Academic Press, pp 233-252

North DW, Balson WE (1985) Risk assessment and acid rain policy: a decision framework that includes uncertainty. In Mandelbaumn P, ed, Proceedings of the conference Acid Rain: Economic Assessment. Plenum Press, New York

NRC (National Research Council) (1983) Risk Assessment in the Federal Government: Managing the Process. National Academy Press, Washington, D.C.

NRC (National Research Council) (1993) Issues in Risk Assessment. Committee on Risk Assessment Methodology, National Research Council. National Academy Press, Washington, D.C.

NRC (National Research Council) (1996) Understanding Risk - Informing Decisions in a Democratic Society. Committee on Risk Assessment Methodology, National
Research Council. National Academy Press, Washington, D.C.

NRC (National Research Council) (2002) Environmental Effects of Transgenic Plants - the Scope and Adequacy of Regulation. Committee on Risk Assessment Methodology, National Research Council, National Academy Press. Washington, D.C.

OGTR (Office of the Gene Technology Regulator) (2001) Risk assessment framework for license applications to the office of the gene technology regulator, Canberra, Australia

OSTP (Office of Science and Technology Policy) (2001) Case Studies of Environmental Regulations for Biotechnology, Executive Office of the President, United States of America, Washington, D.C.

PCT (Pew Charitable Trust) (2002) Three years later: Genetically engineered corn and the monarch butterfly controversy. Issue Brief, Pew Initiative on Food and Biotechnology. Document available free of charge at http:// www.pewtrusts.com

Peterman RM (1990) Statistical power analysis can improve fisheries research and management. Can. J. Fish. Aquat. Sci. 47: 2-15

Power M, McCarty LS (1997) Fallacies in ecological risk assessment practices. Environ. Sci. Technol. 31: 370A-375A

Punt AE, Hilborn R (1997) Fisheries stock assessment and decision analysis: the Bayesian approach. Rev. Fish Biol. Fish. 7: 35-63

Reckhow KH (1994) Importance of Scientific Uncertainty in Decision Making. Environ. Manage. 18: 161-166

Sears MK, Hellmich RL, Stanley-Horn DE, Oberhauser KS, Pleasants JM, Mattila HR, Siegfried BD, Dively GP (2001) Impact of Bt corn pollen on monarch butterfly populations: a risk assessment. PNAS 98: 11937-11942

Strandberg B, Kjellsson G, Lokke H (1998) Hierarchical risk assessment of transgenic plants: proposal for an integrated system. Biosafety J. 4: 21

Strauss HS (1991) Lessons from chemical risk assessment. In Levin MA, Strauss HS, eds, Risk Assessment in Genetic Engineering. McGraw-Hill Inc, New York, pp 297-318

Suter GW II (1990) Uncertainty in Environmental Risk Assessment. In Furstenberg GM, ed, Acting Under Uncertainty: Multidisciplinary Conceptions. Kluwer Academic Publishers, pp 203-230

Suter GW II (1993) Ecological Risk Assessment. Lewis Publishers, Boca Raton

Thompson GG (1992) A Bayesian approach to management advice when stock-recruitment parameters are uncertain. Fish. Bull. 90: 561-573

UNEP (United Nations Environment Program) (1995) UNEP International Technical Guidelines for Safety in Biotechnology

Vatter PA, Bradley SP, Frey Jr SP, Jackson BB (1978) Quantitative Methods in Management: Text and Cases. Richard D. Irwin, Inc., Illinois

Walters CJ (1986) Adaptive Management of Renewable Resources. Blackburn Press 